\title{
The high-mass SFR G23.01-0.41: from the HMC to the VLBI maser kinematics
}

\author{
A. Sanna $*{ }^{a}$ L. Moscadelli, ${ }^{b}$ R. Cesaroni, ${ }^{b}$ C. Goddi, ${ }^{c}$ and G. Surcis ${ }^{c}$ \\ ${ }^{a}$ Max-Planck-Institut für Radioastronomie, Auf dem Hügel 69, 53121 Bonn, Germany \\ ${ }^{b}$ INAF, Osservatorio Astrofisico di Arcetri, Largo E. Fermi 5, 50125 Firenze, Italy \\ c Joint Institute for VLBI in Europe, Postbus 2, 7990 AA Dwingeloo, The Netherlands \\ E-mail: asanna@mpifr-bonn.mpg.de, cesa@arcetri.astro.it, \\ mosca@arcetri.astro.it, goddi@jive.nl, surcis@jive.nl
}

In this proceeding, we review our ongoing studies about the high-mass star-forming region G23.01-0.41. Our understanding of how massive stars grow up by interacting with the interstellar medium is still incomplete and a number of fundamental questions need to be answered. For instance, does a massive protostar accrete mass only from its own core or it does compete for the material of the whole clump? To face up these problems, observations on linear scales matching the sizes of the molecular clumps (0.1-1 pc) need to be combined with a close view of the protostellar surroundings ( $\lesssim 1000 \mathrm{AU}$ ), to establish the presence of multiple sources, their kinematical structures, and the effects of their mutual interaction. G23.01-0.41 is a compelling target for testing different star formation scenarios. It shows a massive bipolar flow from a compact, and hot, dusty core with a rich spectrum of molecular species. All the strongest molecular maser transitions known to date were found to cluster within $2000 \mathrm{AU}$ from the center of the core. VLBI studies of different maser species observed at multiple epochs allow complementary measurements of the 3-dimensional velocity field of gas close to the forming massive star(s). In particular, our European VLBI Network observations of the strong methanol masers at $6.7 \mathrm{GHz}$ have provided unique information of both the velocity field and magnetic field orientation of the gas at the interface between the core and the outflow.

11th European VLBI Network Symposium \& Users Meeting,

October 9-12, 2012

Bordeaux, France

${ }^{*}$ Speaker. 


\section{Introduction}

In the context of star formation, low-mass stars are known to form both in isolation (e.g., in Taurus) and in clusters (e.g., Orion Nebula), whereas high-mass stars $\left(>8 \mathrm{M}_{\odot}\right)$ preferentially form in associations of a few tens to a few thousands of low- and high-mass stars (e.g., [10]). As a consequence, studies of young massive clusters and their molecular surroundings are fundamental to shed light on the first evolutionary phases of O-B stars. On the other hand, disclosing the earliest phases of single high-mass, young stellar objects (YSOs) is still a challenge due to: i) large distances (of a few kpc, typically), ii) heavy obscuration at optical/near-IR frequencies within their natal cocoon, and iii) the short time scales of the formation process. For low-mass forming stars, disks and jets/outflows were recognized to play a major role controlling the accretion of matter onto a protostar, removing the excess of angular momentum, and clearing out the protostellar envelope. One way to proceed is to search for dynamical signatures of disks and outflows toward high-mass YSOs, to test if the process of massive star formation is qualitatively similar to that of low-mass stars (e.g., [3]).

One comprehensive approach is to link together the information obtained from interferometric observations of thermal lines on a sub-parsec scale $(<1 \mathrm{pc})$ with a close view of the protostellar surroundings $(\lesssim 1000 \mathrm{AU})$, obtained from radio continuum observations of ionized gas and Very Long Baseline Interferometry (VLBI) observations of maser lines. To present an overall picture of the high-mass star-forming region (SFR) G23.01-0.41, we outline here the main properties of its hot molecular core (HMC) together with some open questions, which we have recently investigated by means of high angular-resolution, Sub-millimeter Array (SMA) observations and EVN polarimetric measurements of the strong methanol masers in the region.

\section{Star formation activity toward G23.01-0.41}

A few years ago, we have started an intensive observational campaign focused on the active site of massive star formation G23.01-0.41. The overall star-forming complex has possibly been triggered by the close supernova remnant W41 (e.g., Fig. 1 of [5]), at a distance of about $4.6 \mathrm{kpc}$ from the Sun along the Scutum-Centaurus Arm ([2]). The Spitzer IRAC GLIMPSE images of the field reveal a bright, "green fuzzy" clump that stands as a signpost of an intense outflow activity (e.g., Fig. 3 in [1]). At the center of this clump, a rich spectrum of organic molecules such as the rotational lines of methyl-cyanide $\left(\mathrm{CH}_{3} \mathrm{CN}\right)$ hints at the presence of a hot (several tens of Kelvin) and dense (with densities greater than $10^{6} \mathrm{~cm}^{-3}$ ) dusty core, internally heated by the formation of one or more young, massive stellar objects (e.g., Fig. 7 of [4]). The spectral energy distribution from $3 \mu \mathrm{m}$ to $1 \mathrm{~mm}$ of the dusty envelope surrounding the star forming region indicates the presence of a central bright source (with a bolometric luminosity of several $10^{4} \mathrm{~L}_{\odot}$ ) surrounded by a large mass reservoir of about $10^{3} \mathrm{M}_{\odot}$ (Sanna et al., in prep.). A sub-parsec scale view of the emission from the first rotational transitions of the ${ }^{12} \mathrm{CO}$ molecule shows a large amount of gas outflowing from the densest part of the dusty clump, and mainly channeled along the NE-SW direction (e.g., Fig. 6a of [4]). At a scale of a few tens of thousand AU, the continuum emission of dust grains from the compact core, as well as high-density molecular tracers such as ammonia $\left(\mathrm{NH}_{3}\right)$, evidence that dust and gas flatten along a plane perpendicular to the ${ }^{12} \mathrm{CO}$ outflow (see the left panel of Fig. 1). 
The few thousands AU from the center of the HMC harbor all the strongest molecular maser species known to date (e.g., Table 1 and Fig. 1 of [9]), which are useful to unveil the inner gas kinematics around the forming star(s). Single dish spectra of the $22.2 \mathrm{GHz}$ and $6.7 \mathrm{GHz}$ maser transitions from water $\left(\mathrm{H}_{2} \mathrm{O}\right)$ and methanol $\left(\mathrm{CH}_{3} \mathrm{OH}\right)$ gas have a rich amount of strong lines, up to several hundreds of Jy (e.g., Fig. 1 of [8]). At the detail of the VLBI, single spectral features are resolved into individual masing cloudlets of a few AU in size; these cloudlets of molecular gas are associated either with the outflow dynamics (water masers) or with the flattened core dynamics (methanol masers). Water maser emission is spatially distributed along the direction of the large-scale CO outflow, with a number of knots which likely mark the shocked interface between a jet and the surrounding medium (Fig. 5b of [8]). The proper motions of single $\mathrm{H}_{2} \mathrm{O}$ maser cloudlets, measured with multi-epoch Very Long Baseline Array observations ([8]), have directions and amplitudes (several tens of $\mathrm{km} \mathrm{s}^{-1}$ ) indicating that the $\mathrm{H}_{2} \mathrm{O}$ maser jet moves in the right direction and is strong enough to power the large-scale $\mathrm{CO}$ outflow and its more collimated, $\mathrm{SiO}$ counterpart (Sanna et al., in prep.). This picture also accounts for the compact radio continuum emission detected at the center of the core, that would trace ionized gas excited by the passage of the water maser jet ([8]). On the other hand, methanol masers trace a more quiescent environment than water masers, with methanol proper motions revealing an ordered velocity field having the shape of a funnel flowing from the peak position of the dust continuum (see the right panel of Fig. 1). By studying the distribution of the 3-dimensional methanol maser velocities with respect to the jet direction on the sky and its inclination with respect to the line of sight, we propose that the methanol gas undergoes rotation about the jet axis and is simultaneously dragged into the outflow motion along this axis. The outflow cavities (or the surface of a flared disk) in the proximity of a YSO may provide a suitable environment for the excitation of the $6.7 \mathrm{GHz}$ methanol masers. The dust grains, heated by the stellar radiation, reradiate at the right wavelength to pump the $6.7 \mathrm{GHz}$ transition (at an equilibrium temperature around $100-200 \mathrm{~K}$ ), and enhance the gas phase methanol abundance by evaporating methanol-rich ice mantles. To verify our conjectures on the origin of the 6.7 GHz methanol masers, we have conducted complementary observations using the SMA to observe thermal tracers of dense and hot gas at high angular resolution (a few 0.1 arcsec), and the EVN to study the polarized emission of the $6.7 \mathrm{GHz}$ methanol masers. The SMA observations in the $230 \mathrm{GHz}$ band were designed to cover both, high-excitation molecular lines tracing fast-moving gas close to the outflow axis (e.g., ${ }^{12} \mathrm{CO}$ and isotopomers, and $\mathrm{SiO}$ ), and typical tracers of the hot, dense gas close to the $\mathrm{YSO}$ (e.g., $\mathrm{CH}_{3} \mathrm{CN}$ and $\mathrm{CH}_{3} \mathrm{OH}$ lines). Our preliminary analysis of the SMA data reveals that the velocity field of the $\mathrm{CH}_{3} \mathrm{CN}$ gas close to the central object(s) resemble that traced by the methanol masers, with the gas rotating and expanding along the outflow direction (Sanna et al., in prep.). The results of our previous and new EVN observations are summarized in the following section.

\section{The contribution of EVN observations}

We employed most of the EVN antennas, with the addition of the South African antenna Hartebeesthoek to improve on the longest baseline available, to measure: 1) the 3-dimensional velocity field of the $6.7 \mathrm{GHz} \mathrm{CH} \mathrm{CH}_{3} \mathrm{OH}$ maser cloudlets and 2) the orientation of the magnetic field threading the $\mathrm{CH}_{3} \mathrm{OH}$ gas. The EVN observations were designed to image individual centers of 
$\mathrm{CH}_{3} \mathrm{OH}$ maser emission with both a high spectral $\left(<0.1 \mathrm{~km} \mathrm{~s}^{-1}\right)$ and spatial $(\sim 8$ mas round $)$ resolution. These observations were also performed in phase referencing mode, in order to infer the absolute position of the maser distribution with an accuracy of a few milli-arcseconds.

Between 2006 and 2009, we monitored the methanol maser emission in G23.01-0.41 at time intervals of one year under EVN programs EM061 and EM069. Individual cloudlets of methanol maser emission have lifetimes of several years and typical proper motions between about $5-10 \mathrm{~km} \mathrm{~s}^{-1}$ (e.g., Fig. 6 of [7], and [6]). The EVN maps at different epochs allowed us to measure the average velocity vectors of each maser cloudlet with a relative uncertainty of less than $30 \%$, and to sample the 3-dimensional gas kinematics over a radius of about $1000 \mathrm{AU}$ around the center of the HMC ([8]). These observations suggest that the methanol masers trace the inner portion of a massive, rotating toroid extended along the $\mathrm{SW}-\mathrm{NE}$ direction, and excavated along the $\mathrm{NW}-\mathrm{SE}$ direction by a massive outflow (see Fig. 1).

Recent polarization measurements by Surcis et al. [11] have proven the capability of the EVN to detect the few percent linear polarization and Zeeman splitting of the $\mathrm{CH}_{3} \mathrm{OH}$ non-paramagnetic molecule (see the contribution by Surcis et al. in these proceeding series). The high brightness temperature of individual maser spots in G23.01-0.41 (more than half of the 81 cloudlets detected have peak intensities greater than several $\mathrm{Jy} \mathrm{beam}^{-1}$; see Table 4 in [8]) allows us to derive the magnetic field orientation over all the region of maser emission and to compare it with the velocity field traced by the $6.7 \mathrm{GHz} \mathrm{CH} 3 \mathrm{OH}$ masers. In late 2011, the EVN observations of the $6.7 \mathrm{GHz}$ masers in G23.01-0.41 have been repeated in full polarization mode, and with an improved velocity resolution of $0.04 \mathrm{~km} \mathrm{~s}^{-1}$, in order to detect small Zeeman-splitting effects. Our preliminary analysis of the new EVN data shows that the local 3-D magnetic field orientation is not randomly distributed but it does follow two main directions, that of the outflow axis and the elongation axis of the flattened core (Sanna et al., in prep.).

\section{Acknowledgments}

This work was partially funded by the ERC Advanced Investigator Grant GLOSTAR (247078).

\section{References}

[1] E. D. Araya, P. Hofner, W. M. Goss, et al. 2008, ApJS, 178, 330

[2] A. Brunthaler, M. J. Reid, K. M. Menten, et al. 2009, ApJ, 693, 424

[3] R. Cesaroni, D. Galli, G. Lodato, et al. 2007, Protostars and Planets V, 197

[4] R. S. Furuya, R. Cesaroni, S. Takahashi, et al. 2008, ApJ, 673, 363

[5] D. A. Leahy, \& W. W Tian, 2008, AJ, 135, 167

[6] L. Moscadelli, A. Sanna, \& C.Goddi 2011, A\&A, 536, A38

[7] A. Sanna, L. Moscadelli, R. Cesaroni, et al. 2010a, A\&A, 517, A71

[8] A. Sanna, L. Moscadelli, R. Cesaroni, et al. 2010b, A\&A, 517, A78

[9] A. Sanna, L. Moscadelli, R. Cesaroni, \& C. Goddi 2012, IAU Symposium, 287, 396

[10] S. W. Stahler, F. Palla, \& P. T. P. Ho 2000, Protostars and Planets IV, 327

[11] G. Surcis, W. H. T. Vlemmings, R. Dodson, \& H. J. van Langevelde 2009, A\&A, 506, 757 

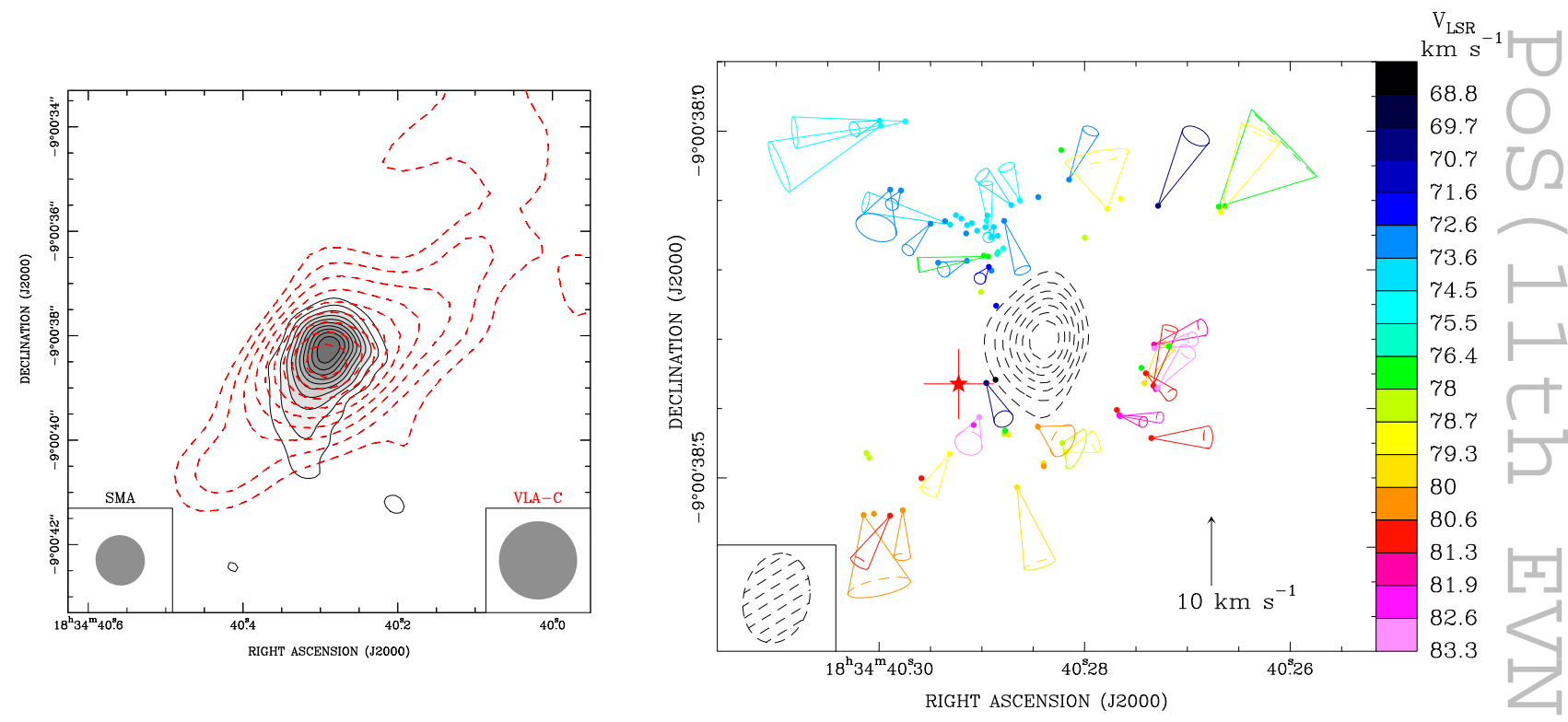

Figure 1: The inner Hot Molecular Core of G23.01-0.41. Left panel: VLA-C map of the $\mathrm{NH}_{3}(3,3)$ line emission (red dashed contours) superposed on a map of the $1.3 \mathrm{~mm}$ dust continuum emission from our new SMA observations (grayscale; Sanna et al., prep.). Contour levels start from $5 \sigma$ by $3 \sigma$ steps for the $1.3 \mathrm{~mm}$ map and from $3 \sigma$ by $1 \sigma$ steps for the $\mathrm{NH}_{3}(3,3)$ map. The SMA and VLA-C synthesized beams are shown in the lower left and right corner, respectively. Right panel: Absolute positions (dots) and proper motions (colored cones) of the $\mathrm{CH}_{3} \mathrm{OH}$ maser cloudlets in $\mathrm{G} 23.01-0.41$. Cones are used to show both the direction and the uncertainty (cone aperture) of individual proper motions with the amplitude scale on the bottom right corner (black arrow). Different colors are used to mark the maser LSR velocities, according to the color scale on the righthand side of the plot, with the green color at the systemic velocity of the HMC. The red star and error bars mark the peak position of the dust continuum within an uncertainty of $1 \sigma$ (Sanna et al., prep.). Dashed contours show the VLA $1.3 \mathrm{~cm}$ continuum emission. Contour levels range from $30 \%$ to $90 \%$ of the peak emission at multiples of $10 \%$, with the restoring beam on the lower left corner ([8]). 